\title{
Clinical and Radiological Outcomes of Modified Phemister Operation with Coracoclavicular Ligament Augmentation Using Suture Anchor for Acute Acromioclavicular Joint Dislocation
}

\author{
Nam Su Cho ${ }^{\varpi}$, Sung Ju Bae, Joong Won Lee, Jeung Hwan Seo, Yong Girl Rhee \\ Shoulder \& Elbow Clinic, Department of Orthopaedic Surgery, College of Medicine, Kyung Hee University, Seoul, Korea
}

Background: Modified Phemister operation has been widely used for the treatment of acute acromioclavicular (AC) joint dislocation. Additionally, the use of suture anchor for coracoclavicular (CC) fixation has been reported to provide CC stability. This study was conducted to evaluate the clinical and radiological results of a modified Phemister operation with CC ligament augmentation using suture anchor for acute AC joint dislocation.

Methods: Seventy-four patients underwent the modified Phemister operation with CC ligament augmentation using suture anchor for acute AC joint dislocation and were followed-up for an average of 12.3 months. The visual analogue scale (VAS), range of motion, Constant score, and Korean shoulder scoring system (KSS) were used for clinical assessment. Acromioclavicular interval (ACl), coracoclavicular distance (CCD), and acromioclavicular distance (ACD) were obtained to evaluate the radiological assessments.

Results: At the last follow-up, the mean VAS Score was 1.7 points, the mean joint range of the forward flexion was $164.6^{\circ}$, external rotation at the side was $61.2^{\circ}$, and internal rotation to the posterior was a level of T12. The mean Constant score and the mean KSS was 82.7 points and 84.2 points, respectively. At the mean $\mathrm{ACI}, \mathrm{CCD}$, and $\mathrm{ACD}$, significant differences were found preoperatively and at the last follow-up. When the $\mathrm{ACl}, \mathrm{CCD}$, and $\mathrm{ACD}$ were compared with the contralateral unaffected shoulder at the last follow-up, the affected shoulders had significantly higher values.

Conclusions: The modified Phemister operation with CC ligament augmentation using suture anchor is clinically and radiologically effective at acute AC joint dislocation.

(Clin Shoulder Elbow 2019;22(2):93-99)

Key Words: Shoulder; Acromioclavicular joint; Dislocation; Modified Phemister; Coracoclavicular ligament augmentation

\section{Introduction}

Acromioclavicular (AC) joint injuries are common, accounting for $9 \%$ to $12 \%$ of all shoulder girdle injuries. ${ }^{1,2)}$ High-grade injuries (Rockwood type IV, V, and VI) of the AC joint are treated surgically, and in the case of type III injuries, indication for surgery is based on the patient's functional demand. ${ }^{3-5)}$ Many operative techniques for acute AC joint injury have been developed over the years, including the Phemister ${ }^{6)}$ or modified Phemister technique, ${ }^{7,8)}$ Bosworth technique, ${ }^{9)}$ Weaver-Dunn technique, ${ }^{10)}$ Wolter plate, conventional hook plate, and locking hook plate fixation. ${ }^{11)}$ Recently, the arthroscopic reduction technique using Tightrope (Arthrex, Naples, FL, USA) ${ }^{12,13)}$ has gained popularity. However, there is no 'gold standard' technique for surgical treatment of AC joint dislocation. ${ }^{3,5}$

Among numerous surgical methods, the relatively simple

Received April 7, 2019. Accepted April 24, 2019.

Correspondence to: Nam Su Cho

Department of Orthopaedic Surgery, Kyung Hee University Hospital at Gangdong, College of Medicine, Kyung Hee University, 892 Dongnamro, Gangdong-gu, Seoul 05278, Korea

Tel: +82-2-440-6154, Fax: +82-2-440-7498, E-mail: nscos1212@empas.com, ORCID: https://orcid.org/0000-0003-1643-1641

IRB approval: Kyung Hee University Hospital at Gangdong (No. KHNMC 2019-04-009).

Financial support: None. Conflict of interests: None. 
modified Phemister technique with Kirschner wire (K-wire) is a surgical technique that has commonly been used for the last few decades in acute AC joint dislocation, and many studies have reported clinical results of this technique. ${ }^{14-16)}$ However, there are several complications associated with this technique owing to the long fixation period of K-wire and it does not solve coracoclavicular ligament injuries. ${ }^{14,17,18)}$

Recently, the use of suture anchors has been described for the treatment of AC joint injuries. These devices offer the potential advantages of technical ease of use and reduced risk of neurovascular injury, as well as improved pullout strength, which have led to their widespread use in treatment. ${ }^{19,20)}$ However, only a few studies have reported the clinical and radiologic outcomes of coracoclavicular ligament augmentation using suture anchor devices for treatment of acute AC joint injury. ${ }^{21)}$

Therefore, this study was conducted to evaluate the clinical and radiological outcomes of modified Phemister operation and additional coracoclavicular ligament augmentation using suture anchors for acute AC joint dislocation. In addition, the clinical and radiological outcomes were evaluated retrospectively. We hypothesized that modified Phemister operation and coracoclavicular ligament augmentation using suture anchors provide satisfactory restoration of shoulder function and radiological outcomes.

\section{Methods}

\section{Patient Selection}

From July 2007 to March 2018, a consecutive series of 74 patients receiving modified Phemister operation with coracoclavicular ligament augmentation using suture anchor for acute AC joint dislocation were studied retrospectively (Table 1).

Inclusion criteria of patients were (1) radiographically confirmed, acute Rockwood type III or higher AC dislocation (2) underwent surgery within 2 weeks. Exclusion criteria were (1) subacute (more than two weeks since injury) or chronic AC joint dislocation, (2) previous history of surgery on the affected shoul-

Table 1. Patients Demographics

\begin{tabular}{|lc|}
\hline \multicolumn{1}{|c|}{ Variable } & Patient $(\mathrm{n}=74)$ \\
\hline Sex (male/female) & $72 / 2$ \\
\hline Age (yr) & $48.0 \pm 12.4(17-68)$ \\
\hline Injured side (right/left) & $39 / 35$ \\
\hline Follow-up (mo) & $12.34 \pm 18.56$ \\
\hline Grade of acromioclavicular dislocation & \\
\hline Type III & $13(17.6)$ \\
Type V & $61(82.4)$ \\
\hline
\end{tabular}

Values are presented as number only, mean \pm standard deviation (range), or number (\%). der, (3) concomitant fracture around the ipsilateral shoulder.

A total of 72 males and two females with a mean age of 48.0 years (range, 17-68 years) were included in this study. The mean duration of follow-up from surgery was 12.3 months (range, 8-24 months). According to Rockwood's classification, ${ }^{22)}$ there were 13 type III lesions (17.6\%) and 61 type V lesions (82.4\%). All patients underwent surgery within a mean of four days (range, 1-9 days). All patients in our series had coracoclavicular ligament injuries. Patients with type $\mathrm{V}$ lesions were immediately addressed to surgical treatment, while indication for surgery for patients with type III lesions was determined based on the patient's functional demand. All patients were operated on by the same surgeon and provided informed consent to be included in the study.

\section{Surgical Technique}

Under general anesthesia, all patients underwent surgery in the $30^{\circ}$ beach chair position on an ordinary operating table. A vertical incision was made just lateral to the coracoid along the Langer lines extending from the posterior edge of the AC joint to the coracoid process. The lateral end of the clavicle, the acromion, and the AC joint were exposed. The attachment of the torn coracoclavicular ligament was usually seen at the 'tip' of the coracoid process. One suture anchor (Super Revo; Conmed, Largo, FL, USA) was placed into the base of the coracoid process, and the sutures were pulled manually several times to ensure appropriate seating and secure fixation. After the anchor was deployed, two drill holes were made across the bone in the insertion site of the coracoclavicular ligament in the clavicle, and looped wires were passed through the created holes. The clavicle was then reduced by the surgical assistant and confirmed by fluoroscopy. To maintain the obtained reduction, K-wires were trans-articularly inserted through the AC joint under fluoroscopic guidance and bent to prevent forward migration. When a satisfactory reduction was achieved, the joint was stabilized with sutures passing through the created holes and sutures were tied over the clavicle (Fig. 1, 2). Postoperatively, the treated arm was supported with a sling. K-wires were removed at 8 weeks after AC joint stabilization.

\section{Clinical Assessment}

Postoperative clinical evaluations were performed regularly on an outpatient basis (at 4 weeks, 6 weeks, 2 months, and 6 months following surgery and at the last follow-up). The results of the evaluations at the last follow-up were analyzed. At the time of follow-up, all patients were evaluated using the visual analogue scale (VAS) for subjective pain. Postoperative shoulder ranges of motion (ROM), including forward flexion (FF), external rotation at the side (ERs), and internal rotation to the back (IRp) were assessed. The Constant score and Korean shoulder scoring system (KSS) were used for clinical assessments. ${ }^{23,24)}$ 

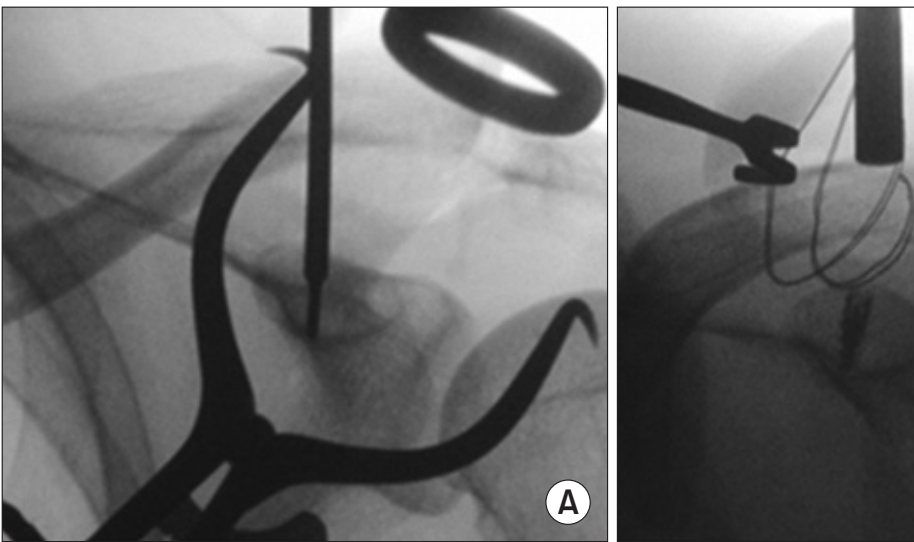

(A)
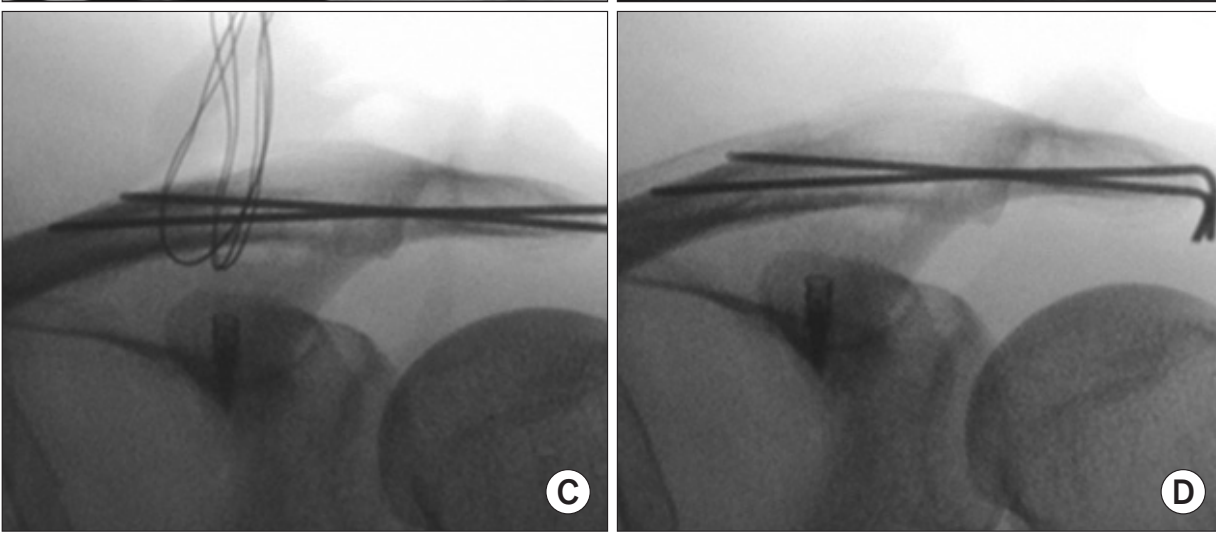

(D)
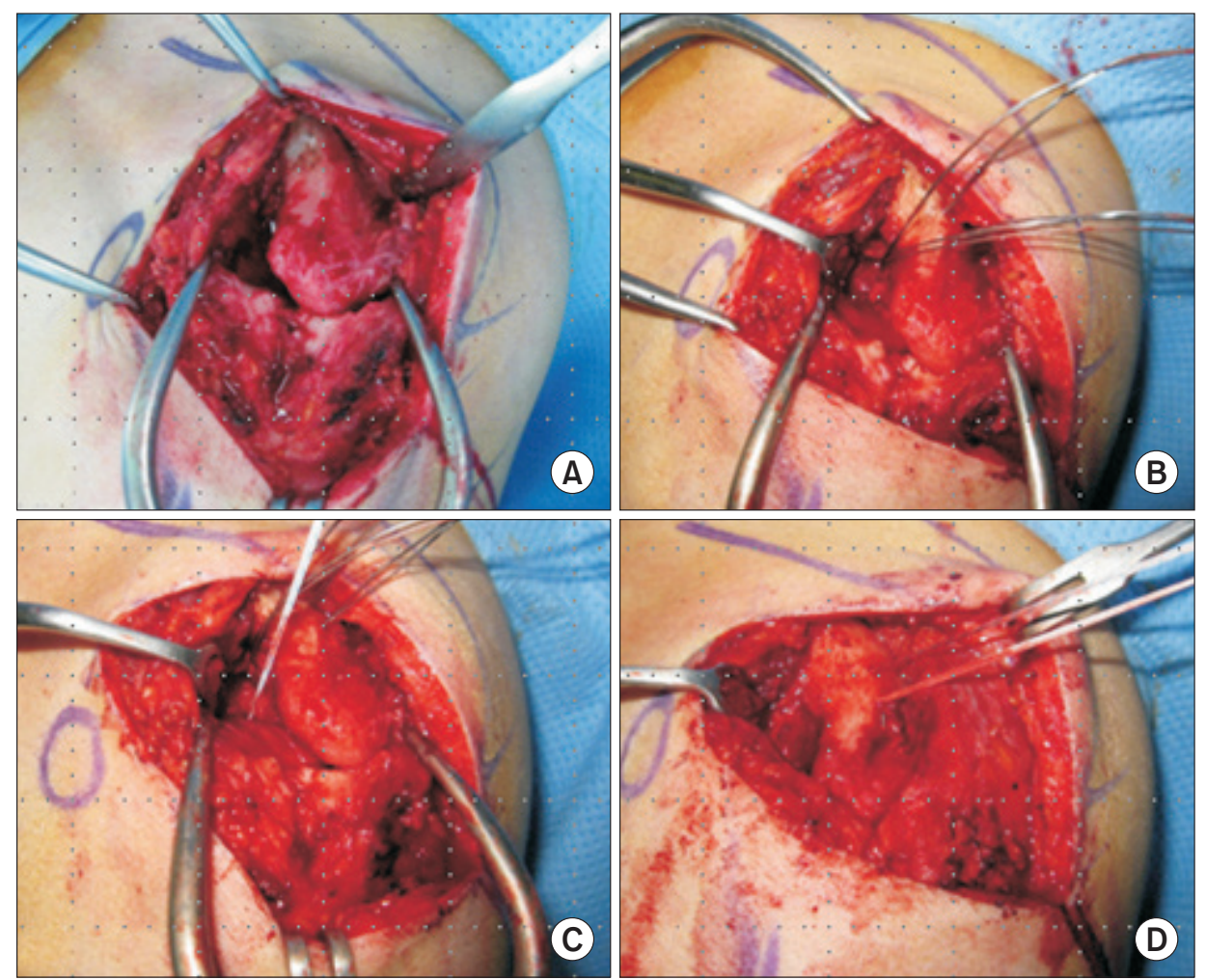

Fig. 1. Intraoperative radiographs. (A) Suture anchor was fixed at the original site of the coracoclavicular ligament in the coracoid process. (B) Looped wires were passed through the holes created in the insertion site of coracoclavicular ligament in the clavicle. (C) Reduction of acromioclavicular joint with two Kirschner wires transfixation. (D) Sutures were tied over the clavicle and the radiograph shows anatomical reduction of the acromioclavicular joint.
Fig. 2. Intraoperatve photographs. (A) The lateral end of the clavicle, the acromion, and the acromioclavicular joint were exposed. (B) The looped wires were passed through the two created holes. (C) One suture anchor was placed into the base of the coracoid process. (D) When a satisfactory reduction and $\mathrm{K}$-wires fixation were achieved, the joint was stabilized with sutures passing through the created holes. 


\section{Radiological Assessment}

Anteroposterior radiographs of the affected and contralateral unaffected AC joint in neutral rotation made with the patient in a standing position were obtained immediately postoperatively and at each follow-up examination. Acromioclavicular interval (ACl) was defined as the perpendicular distance between the clavicle distal end and acromion, while coracoclavicular distance (CCD) was defined as the perpendicular distance between the upper border of the coracoids process and the inferior cortex of the clavicle, and acromioclavicular distance (ACD) was defined as the perpendicular distance between the line passing the upper border of the acromion and the line parallel to the upper border of the lateral part of the clavicle. All radiographs were analyzed by two authors who reached a consensus. $p$-values of less than 0.05 were considered significant.

\section{Results}

\section{Clinical Outcomes}

The clinical and radiological outcomes are presented in Table 2. The VAS, ROM, Constant, and KSS score were evaluated as clinical factors at the last follow-up. The mean VAS score while resting was $1.7 \pm 0.5$ points and that while active was $2.4 \pm$ 1.2 points. The mean joint range of the FF was $164.6^{\circ} \pm 21.5^{\circ}$, while ERs was $61.2^{\circ} \pm 18.7^{\circ}$, and IRp was $\mathrm{T} 12.9 \pm 2.6$. The mean Constant score was $82.7 \pm 6.6$ points, while the mean KSS score was $84.2 \pm 3.6$ points. All cases achieved a satisfactory outcome.

\section{Radiologic Outcomes}

Significant differences were observed between the average $\mathrm{ACl}, \mathrm{CCD}$, and $\mathrm{ACD}$ in the preoperative stage and that on the

Table 2. Clinical Outcomes of Modified Phemister Operation with Coracoclavicular Ligament Augmentation Using Suture Anchor

\begin{tabular}{|cc|}
\hline \multicolumn{1}{|c|}{ Variable } & Postoperative outcome \\
\hline VAS pain & $1.7 \pm 0.5$ \\
\hline At rest & $2.4 \pm 1.2$ \\
\hline Active & \\
\hline Range of motion & $164.6 \pm 21.5$ \\
\hline FF $\left(^{\circ}\right)$ & $61.2 \pm 18.7$ \\
\hline ERs $\left(^{\circ}\right)$ & T12.9 \pm 2.6 \\
\hline IRp & $84.2 \pm 3.6$ \\
\hline KSS score & $82.7 \pm 6.6$ \\
\hline Constant score & \\
\hline
\end{tabular}

Values are presented as the mean \pm standard deviation.

VAS: visual analogue scale, FF: forward flexion, ERs: external rotation at the side, IRp: internal rotation to the posterior, KSS: Korean shoulder scoring system. last follow-up radiographs of the affected shoulder (Table 3). The average $\mathrm{ACl}, \mathrm{CCD}$, and $\mathrm{ACD}$ were significantly reduced on the last follow-up. The average decrease of $\mathrm{ACl}$ was $3.1 \mathrm{~mm}$ $(9.03 \pm 3.02 \mathrm{~mm}$ vs. $5.89 \pm 2.58 \mathrm{~mm}, p<0.001)$, while that of CCD was $5.8 \mathrm{~mm}(17.59 \pm 4.50 \mathrm{~mm}$ vs. $11.86 \pm 3.83 \mathrm{~mm}$, $p<0.001)$, and that of ACD was $7.1 \mathrm{~mm}(17.66 \pm 5.70 \mathrm{~mm}$ vs. $10.58 \pm 3.91 \mathrm{~mm}, p<0.001)$ based on evaluation of the radiographs.

Comparison of the $\mathrm{ACl}, \mathrm{CCD}$, and $\mathrm{ACD}$ values of the contralateral unaffected shoulder and affected shoulder at the last follow-up revealed that the affected shoulders had significantly higher average $\mathrm{ACl}(3.21 \pm 1.67 \mathrm{~mm}$ vs. $5.89 \pm 2.58 \mathrm{~mm}$, $p<0.001), \operatorname{CCD}(8.11 \pm 2.83 \mathrm{~mm}$ vs. $11.86 \pm 3.83 \mathrm{~mm}$, $p<0.001)$, and $\operatorname{ACD}(7.73 \pm 3.01 \mathrm{~mm}$ vs. $10.58 \pm 3.91 \mathrm{~mm}$, $p=0.004$ ) values (Table 4).

\section{Complications}

There were four cases $(5.4 \%)$ of skin irritation, migration of the K-wire occurred in three $(4.1 \%)$ patients and breakage occurred in eight patients (10.8\%). When K-wire migration or breakage occurred, hardware was removed after AC joint stabilization was confirmed. These patients did not complain of pain after hardware removal. At the time of K-wire removal, we had no case of immediate secondary re-dislocation of the AC joint. Follow-up radiographs showed AC osteoarthritis in two patients (2.7\%). No patients had intraoperative or postoperative clavicular or coracoid fracture, infection, or revision surgery.

Table 3. Radiologic Outcomes of Modified Phemister Operation with Coracoclavicular Ligament Augmentation Using Suture Anchor

\begin{tabular}{cccc}
\hline Variable & Preoperative $(\mathrm{mm})$ & Last follow-up $(\mathrm{mm})$ & $p$-value \\
\hline ACI & $9.03 \pm 3.02$ & $5.89 \pm 2.58$ & $<0.001$ \\
CCD & $17.59 \pm 4.50$ & $11.86 \pm 3.83$ & $<0.001$ \\
ACD & $17.66 \pm 5.70$ & $10.58 \pm 3.91$ & $<0.001$ \\
\hline
\end{tabular}

Values are presented as the mean \pm standard deviation.

ACI: acromioclavicular interval, CCD: coracoclavicular distance, ACD: acromioclavicular distance.

${ }^{*}$ Statistically significant $(p<0.05)$.

Table 4. Radiological Outcomes in Comparison to Unaffected Side

\begin{tabular}{cccc}
\hline Variable & Unaffected side $(\mathrm{mm})$ & Last follow-up $(\mathrm{mm})$ & $p^{\text {-value }}$ \\
\hline ACI & $3.21 \pm 1.67$ & $5.89 \pm 2.58$ & $<0.001$ \\
CCD & $8.11 \pm 2.83$ & $11.86 \pm 3.83$ & $<0.001$ \\
ACD & $7.73 \pm 3.01$ & $10.58 \pm 3.91$ & 0.004 \\
\hline
\end{tabular}

Values are presented as the mean \pm standard deviation.

ACI: acromioclavicular interval, CCD: coracoclavicular distance, ACD: acromioclavicular distance.

${ }^{\star}$ Statistically significant $(p<0.05)$. 


\section{Discussion}

The principal finding of the present study was that modified Phemister operation using augmentation of the coracoclavicular ligament by suture anchor for the treatment of acute AC joint dislocation successfully restored the stability of the AC joint. Satisfactory clinical and radiologic outcomes were simultaneously achieved in this study. At the final follow-up, the mean VAS score was $1.7 \pm 0.5$ points, the Constant score was $82.7 \pm 6.6$ points, and the KSS score was $84.2 \pm 3.6$ points. An acceptable joint alignment was achieved in all the patients after surgery. Furthermore, the authors found no problems achieving AC joint stabilization by this technique. In addition, the technique was simple with low hardware costs.

Over the last decade, numerous surgical procedures have been described for the management of acute AC joint dislocation. ${ }^{4,5)}$ Despite the use of various methods of surgical treatment of acute AC joint dislocations such as K-wire, hook-plates, screws, synthetic sling materials, and ligament reconstruction, no technique has been shown to be clinically superior to another. ${ }^{4,10,25-27)}$ Among these methods, the modified Phemister technique with K-wire is relatively simple, fast, and inexpensive. Additionally, the use of a suture anchor offers the advantage of quick fixation of the coracoclavicular ligament to bone while minimizing extensive soft tissue exposure. ${ }^{20,28}$

Rolf et al. ${ }^{29)}$ reported the outcomes of 29 patients treated with a modified Phemister technique that included augmentation of the coracoclavicular ligaments with polydioxanone suture. Specifically, an additional polydioxanone cord was passed around the base of the coracoids and formed into a figure8-shaped sling through a hole drilled in the lateral clavicle. After a follow-up of 53 months (range, 20 to 92 months), the authors showed clinical results statistically favoring the early treatment of injuries. Corella et al. ${ }^{30)}$ reviewed the outcomes of type III and type V AC dislocations treated using the Phemister technique and found good results in the immediate postoperative period, but long-term relapses in $66 \%$ of the cases, despite a high level of subjective satisfaction reported by patients. Moon et al. ${ }^{21)}$ conducted a retrospective study of 14 patients who underwent a modified Phemister procedure with a suture anchor added for augmentation of the conoid ligament in cases of acute dislocation of the AC joint. At the mean follow-up (14 months), functional results were good/excellent with a Constant score of 90.8 and KSS score of 91. In our study, we observed good clinical and functional results at the follow-up examination with respect to the ROM, strength and pain discomfort. Moreover, the Constant and KSS scores showed global satisfaction with the procedure.

Rhee et al ${ }^{16)}$ reported good radiologic outcome in a retrospective study that compared the K-wire transfixation group and the locking hook plate group. Overall, they showed that the Kwire transfixation group had an average increase in $\mathrm{ACl}$ of 2.2 $\mathrm{mm}$, CCD of $2.0 \mathrm{~mm}$, and ACD of $1.8 \mathrm{~mm}$ upon comparison of the contralateral unaffected shoulder and affected shoulder at the last follow-up. Verdano et al. ${ }^{31)}$ used a modified Phemister technique that combined loop augmentation, coracoclavicular ligaments repair and transarticular stabilization with K-wire for acute AC joint dislocation. In their study, at the time of radiological follow-up, three patients maintained the reduction (21.4\%), while nine $(64.3 \%)$ showed a partial recurrence of the dislocation $(<3 \mathrm{~mm})$ and two (14.3\%) showed complete re-dislocation. Kim and Chun ${ }^{32)}$ compared use of tans-acromial fixation and the $\mathrm{AO}$ hook plate for the treatment of acute AC joint dislocation and found that the K-wire trans-acromial fixation group had a significantly greater $\mathrm{CCD}$ than the $\mathrm{AO}$ locking hook plate group. In the current study, the average $\mathrm{ACl}, \mathrm{CCD}$, and $\mathrm{ACD}$ was significantly reduced at the last follow-up and all patients maintained an acceptable reduction of the $\mathrm{AC}$ joint.

Leidel et al. ${ }^{15)}$ described K-wire migration in $4 \%(n=3)$ and secondary dislocation of the AC joint in $11 \%(n=8)$ of cases after wire removal. None of the 70 patients who underwent surgery for type III injuries became infected. Mayr et al. ${ }^{33)}$ reported a loss of reduction rate of $28 \%$, with a less satisfactory outcome in patients who underwent the modified Phemister procedure. Calvo et al. ${ }^{34)}$ used the modified Phemister technique in 32 surgically treated type III AC dislocations and found that only $50 \%$ of the AC joints were reduced in long-term follow-up, with a significantly higher incidence of osteoarthritis (81.2\%) and coracoclavicular ligament ossification (59.4\%). In the present study, all Kwires were inserted bicortically to provide more stable fixation and they were bent to prevent forward migration. Moreover, efforts were made to provide coracoclavicular ligament augmentation, which helped reduce the incidence of complete failure or re-dislocation.

It should be noted that this study has several limitations. First, being retrospective in nature, our study has limitations similar to other retrospective studies. Second, the follow-up period was relatively short and the incidence of arthritis in the AC joint would be different in long-term follow-up. However, the strengths of this study include that the procedure was relatively simple, fast, and inexpensive; and all operations were performed by a single surgeon.

\section{Conclusion}

The modified Phemister operation with coracoclavicular ligament augmentation using a suture anchor led to satisfactory clinical and radiological outcomes and was found to be safe, showing a low complication rate in acute dislocations of the AC joint. This surgical technique also has a low learning curve procedure with low hardware costs. Our data suggest that the modified Phemister procedure for the surgical treatment of acute AC joint dislocations is a reliable technique for restoration of shoulder girdle. 


\section{References}

1. Bontempo NA, Mazzocca AD. Biomechanics and treatment of acromioclavicular and sternoclavicular joint injuries. Br J Sports Med. 2010;44(5):361-9. doi: 10.1136/bjsm.2009.059295.

2. Tauber M. Management of acute acromioclavicular joint dislocations: current concepts. Arch Orthop Trauma Surg. 2013;133:985-95. doi: 10.1007/s00402-013-1748-z.

3. Mazzocca AD, Arciero RA, Bicos J. Evaluation and treatment of acromioclavicular joint injuries. Am J Sports Med. 2007;35(2):316-29. doi: 10.1177/0363546506298022.

4. Epstein D, Day M, Rokito A. Current concepts in the surgical management of acromioclavicular joint injuries. Bull NYU Hosp Jt Dis. 2012;70(1):11-24.

5. Beitzel K, Cote MP, Apostolakos J, et al. Current concepts in the treatment of acromioclavicular joint dislocations. Arthroscopy. 2013;29(2):387-97. doi: 10.1016/j.arthro.2012.11.023.

6. Phemister DB. The treatment of dislocation of the acromioclavicular joint by open reduction and threaded-wire fixation. J Bone Joint Surg. 1942;24(1):166-8.

7. Winkler H, Schlamp D, Wentzensen A. [Treatment of acromioclavicular joint dislocation by tension band and ligament suture]. Aktuelle Traumatol. 1994;24(4):133-9. German.

8. García NM, Bermejo FT. Modified Phemister procedure for the treatment of type III Rockwood acromioclavicular joint dislocation. Results after five-years follow-up. Rev Esp Cir Ortop Traumatol. 2010;54(5):294-300. doi: 10.1016/S19888856(10)70249-2.

9. Bosworth BM. Acromioclavicular separation: new method of repair. Surg Gynecol Obstet. 1974;76:866-71.

10. Weaver JK, Dunn HK. Treatment of acromioclavicular injuries, especially complete acromioclavicular separation. J Bone Joint Surg Am. 1972;54(6):1187-94.

11. Balser D. Eine neue methode zur operativen behandlung der akromioklavikulären luxation. Chir Prax. 1976;24:275.

12. Horst K, Dienstknecht T, Christoph Pape H. Surgical treatment of acromioclavicular joint separation. OP J. 2013;29(1):24-30. doi: 10.1055/s-0032-1328234.

13. Salzmann GM, Walz L, Schoettle PB, Imhoff AB. Arthroscopic anatomical reconstruction of the acromioclavicular joint. Acta Orthop Belg. 2008;74(3):397-400.

14. Chun $\mathrm{CH}$, Lee SH, Lee BC, Cho YW. The surgical treatment of acromioclavicular joint dislocation using modified phemister and modified Weaver-Dunn operation. Clin Shoulder Elbow. 1998;1(2):180-5.

15. Leidel BA, Braunstein V, Kirchhoff C, Pilotto S, Mutschler W, Biberthaler P. Consistency of long-term outcome of acute Rockwood grade III acromioclavicular joint separations after K-wire transfixation. J Trauma. 2009;66(6):1666-71. doi: 10.1097/TA.0b013e31818c1455.

16. Rhee YG, Park JG, Cho NS, Song WJ. Clinical and radiologic outcomes of acute acromioclavicular joint dislocation: comparison of Kirschner's wire transfixation and locking hook plate fixation. Clin Shoulder Elbow. 2014;17(4):159-65. doi: 10.5397/cise.2014.17.4.159.

17. Larsen E, Bjerg-Nielsen A, Christensen P. Conservative or surgical treatment of acromioclavicular dislocation. A prospective, controlled, randomized study. J Bone Joint Surg Am. 1986;68(4):552-5.

18. Lyons FA, Rockwood CA Jr. Migration of pins used in operations on the shoulder. J Bone Joint Surg Am. 1990;72(8):12627.

19. Breslow MJ, Jazrawi LM, Bernstein AD, Kummer FJ, Rokito AS. Treatment of acromioclavicular joint separation: suture or suture anchors? J Shoulder Elbow Surg. 2002;11(3):225-9. doi: 10.1067/mse.2002.123904.

20. Su EP, Vargas JH 3rd, Boynton MD. Using suture anchors for coracoclavicular fixation in treatment of complete acromioclavicular separation. Am J Orthop (Belle Mead NJ). 2004; 33(5):256-7.

21. Moon GH, Nam IH, Lee YH, Kim KC, Lee JH, Ahn GY. The modified phemister operation with the suture anchor added for the augmentation of conoid ligament in acute acromioclavicular dislocation. Clin Shoulder Elbow. 2010;13(1):34-9. doi: 10.5397/CiSE.2010.13.1.034.

22. Rockwood C, Williams G, Young D. Disorders of the acromioclavicular joint. In: Rockwood CA, ed. The shoulder. 3rd ed. Philadelphia: Saunders; 2004. 521-95.

23. Constant CR, Murley AH. A clinical method of functional assessment of the shoulder. Clin Orthop Relat Res. 1987;(214):160-4.

24. Tae SK, Rhee YG, Park TS, et al. The development and validation of an appraisal method for rotator cuff disorders: the Korean Shoulder Scoring System. J Shoulder Elbow Surg. 2009;18(5):689-96. doi: 10.1016/j.jse.2008.11.019.

25. Lizaur A, Sanz-Reig J, Gonzalez-Parreño S. Long-term results of the surgical treatment of type III acromioclavicular dislocations: an update of a previous report. J Bone Joint Surg Br. 2011;93(8):1088-92. doi: 10.1302/0301-620X.93B8.26775.

26. Fraser-Moodie JA, Shortt NL, Robinson CM. Injuries to the acromioclavicular joint. J Bone Joint Surg Br. 2008;90(6):697707. doi: 10.1302/0301-620X.90B6.20704.

27. Tomlinson DP, Altchek DW, Davila J, Cordasco FA. A modified technique of arthroscopically assisted AC joint reconstruction and preliminary results. Clin Orthop Relat Res. 2008;466(3):639-45. doi: 10.1007/s11999-007-0085-3.

28. Choi SW, Lee TJ, Moon KH, Cho KJ, Lee SY. Minimally invasive coracoclavicular stabilization with suture anchors for acute acromioclavicular dislocation. Am J Sports Med. 2008;36(5):9615. doi: 10.1177/0363546507312643.

29. Rolf O, Hann von Weyhern A, Ewers A, Boehm TD, Gohlke F. Acromioclavicular dislocation Rockwood III-V: results of early 
versus delayed surgical treatment. Arch Orthop Trauma Surg. 2008;128(10):1153-7. doi: 10.1007/s00402-007-0524-3.

30. Corella F, Ortiz A, Ló D, Ocampos M, Calvo JA, Vaquero J. [Surgical treatment of acromioclavicular dislocations with the Phemister method: the long-term results]. Patol Apar Locomot. 2006;4:157-66. Spanish.

31. Verdano MA, Pellegrini A, Zanelli M, Paterlini M, Ceccarelli F. Modified Phemister procedure for the surgical treatment of Rockwood types III, IV, V acute acromioclavicular joint dislocation. Musculoskelet Surg. 2012;96(3):213-22. doi: 10.1007/ s12306-012-0221-4.
32. Kim YJ, Chun YM. Treatment of acute acromioclavicular joint dislocation: Kirschner's wire trans-acromial fixation versus AO locking hook plate fixation. Clin Shoulder Elbow. 2016;19(3):149-154. doi: 10.5397/cise.2016.19.3.149.

33. Mayr E, Braun W, Eber W, Rüter A. [Treatment of acromioclavicular joint separations. Central Kirschner-wire and PDSaugmentation]. Unfallchirurg. 1999;102(4):278-86. German.

34. Calvo E, López-Franco M, Arribas IM. Clinical and radiologic outcomes of surgical and conservative treatment of type III acromioclavicular joint injury. J Shoulder Elbow Surg. 2006;15(3):300-5. doi: 10.1016/j.jse.2005.10.006. 